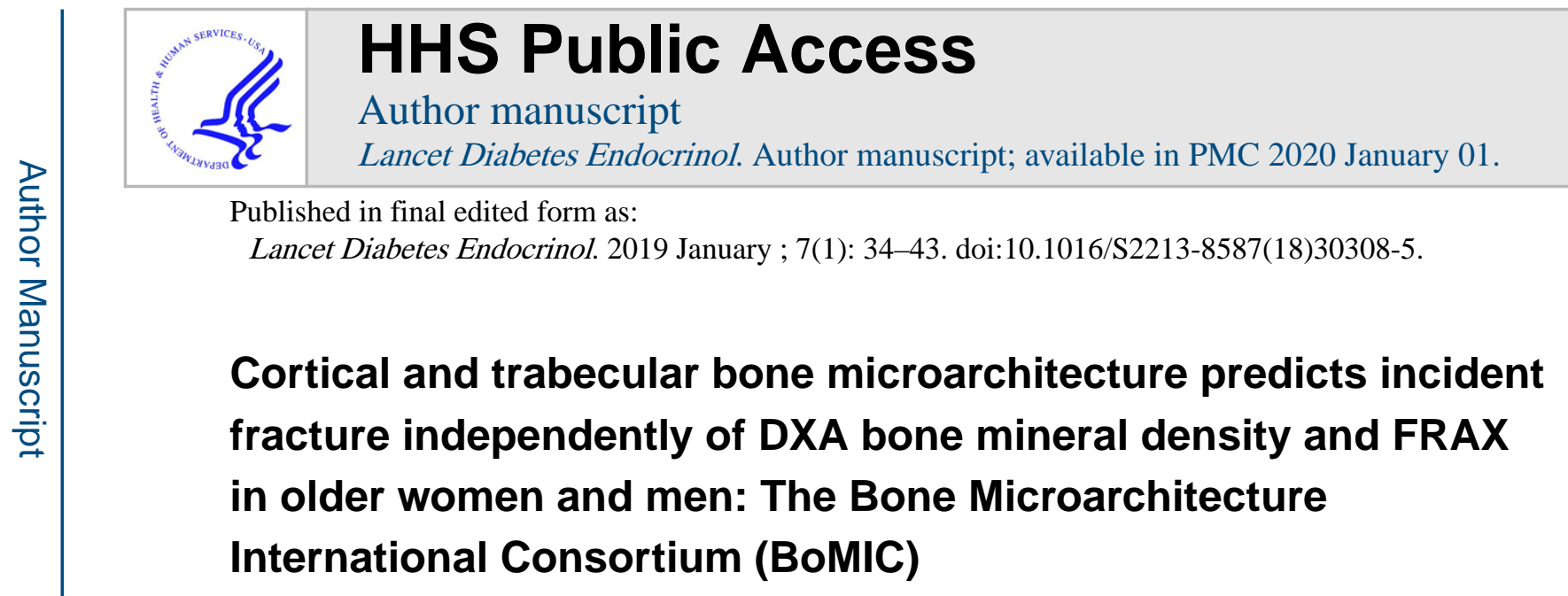

\author{
Samelson Elizabeth J., \\ Institute for Aging Research, Hebrew SeniorLife, Harvard Medical School; Boston, MA
}

Kerry E. Broe, Institute for Aging Research, Hebrew SeniorLife, Boston, MA

Hanfei Xu, Department of Biostatistics, Boston University School of Public Health; Boston, MA

Laiji Yang, Institute for Aging Research, Hebrew SeniorLife, Harvard Medical School, Boston, MA

Steven Boyd,

McCaig Institute for Bone and Joint Health, University of Calgary; Calgary, Alberta, Canad

Emmanuel Biver,

Division of Bone Diseases, Geneva University Hospitals and Faculty of Medicine; University of Geneva, Switzerland

Pawel Szulc, INSERM UMR1033, Université de Lyon, Hôpital Edouard Herriot; Lyon, France

\footnotetext{
Publisher's Disclaimer: This is a PDF file of an unedited manuscript that has been accepted for publication. As a service to our customers we are providing this early version of the manuscript. The manuscript will undergo copyediting, typesetting, and review of the resulting proof before it is published in its final citable form. Please note that during the production process errors may be discovered which could affect the content, and all legal disclaimers that apply to the journal pertain.

Authors' roles: Study design: EJS, MLB, SD, DPK. Study conduct: EJS, MLB, SD, DPK. Data collection: All authors. Data analysis: EJS, MLB, SD, DPK, HX. Data interpretation: EJS, MLB, SD, DPK, EB, SB, CO, PS. Drafting manuscript: EJS and MLB. Revising manuscript content: EJS, MLB, SD, DPK, EB, SB, CO, PS. Approving final version of manuscript: All authors. EJS takes responsibility for the integrity of the data analysis.

The authors acknowledge Alyssa B. Dufour, PhD, for providing statistical expertise.

Declaration of Interests

RR: personal fees from Radius Health, Danone, Pfizer, Sandoz, and CNIEL, outside the submitted work. DH: grants and personal fees from Amgen, grants from Pure North S'Energy Foundation, outside the submitted work. RC: grant and personal fees from Amgen, personal fees from UCB, BMS, Pfizer, grants and personal fees from Merck, personal fees from Abbvie, personal fees from Sanoz, personal fees from Lilly, grants and personal fees from Chugai, outside the submitted work. SB: co-founder of Numerics 88 Solutions Inc. that provides an estimate of bone strength from HR-pQCT images. MLB: grants from National Institutes of Health during the conduct of the study. EB: grants from Geneva University Hospitals and Faculty of Medicine Clinical Research Centre, grants from Geneva University Hospitals Private Foundation during the conduct of the study. JA: grants and personal fees from Amgen, grants and personal fees from Eli Lilly, grants and personal fees from Merck, grants from Actavis, personal fees from Agnovis, outside the submitted work. ML: personal fees from Amgen, Lilly, Meda, Radius Health, Renapharma, Consilient Health, UCB Pharma, outside the submitted work. BVB: Personal fees from Scanco Medical AG, outside submitted work. DPK: Personal fees from Springer, outside submitted work.

All other authors declare no competing interests.
} 
Jonathan Adachi,

Department of Medicine, Michael G. DeGroote School of Medicine, St Joseph's Healthcare -

McMaster University ; Hamilton, Ontario, Canada

Shreyasee Amin,

Mayo Clinic College of Medicine and Science, Rochester, MN

Elizabeth Atkinson,

Mayo Clinic College of Medicine and Science, Rochester, MN

Claudie Berger,

Research Institute of the McGill University Health Centre; Montreal, Quebec, Canada

Lauren Burt,

McCaig Institute for Bone and Joint Health, University of Calgary; Calgary, Alberta, Canada

Roland Chapurlat,

INSERM UMR1033, Université de Lyon, Hôpital Edouard Herriot; Lyon, France

Thierry Chevalley,

Division of Bone Diseases, Geneva University Hospitals and Faculty of Medicine; Geneva,

Switzerland

\section{Serge Ferrari,}

Division of Bone Diseases, Geneva University Hospitals and Faculty of Medicine; Geneva,

Switzerland

David Goltzman,

Departments of Medicine, McGill University and McGill University Health Centre; Montreal, Quebec, Canada

David Hanley,

McCaig Institute for Bone \& Joint Health, University of Calgary; Calgary, Alberta, Canada

Marian T. Hannan,

Institute for Aging Research, Hebrew SeniorLife, Harvard Medical School; Boston, MA

Sundeep Khosla,

Mayo Clinic College of Medicine and Science, Rochester, MN

Ching-Ti Liu,

Department of Biostatistics, Boston University School of Public Health, Boston, MA

Mattias Lorentzon,

Geriatric Medicine and Centre for Bone and Arthritis Research, Institute of Medicine, University of Gothenburg; Gothenburg, Sweden

Dan Mellstrom,

Geriatric Medicine and Centre for Bone and Arthritis Research, Institute of Medicine, Sahlgrenska Academy, University of Gothenburg; Gothenburg, Sweden

Blandine Merle,

INSERM UMR1033, Université de Lyon, Hôpital Edouard Herriot; Lyon, France 
Maria Nethander,

Bioinformatics Core Facility and Centre for Bone and Arthritis Research, Institute of Medicine, Sahlgrenska Academy, University of Gothenburg, Gothenburg, Sweden

\section{René Rizzoli,}

Division of Bone Diseases, Geneva University Hospitals and Faculty of Medicine; Geneva, Switzerland

Elisabeth Sornay-Rendu,

INSERM UMR1033, Université de Lyon, Hôpital Edouard Herriot; Lyon, France

\section{Bert Van Rietbergen,}

Department of Biomedical Engineering, Eindhoven University of Technology; Eindhoven, The Netherlands

Daniel Sundh,

Geriatric Medicine and Centre for Bone and Arthritis Research, Institute of Medicine, University of Gothenburg; Gothenburg, Sweden

\section{Andy Kin On Wong,}

Joint Department of Medical Imaging, University Health Network, Toronto, Ontario, Canada

Claes Ohlsson",

Centre for Bone and Arthritis Research, Institute of Medicine, Sahlgrenska Academy, University of Gothenburg, Gothenburg, Sweden

Serkalem Demissie",

Department of Biostatistics, Boston University School of Public Health; Boston, MA

Douglas P. Kiel ${ }^{\#}$, and Institute for Aging Research, Hebrew SeniorLife, Harvard Medical School; Boston, MA

\section{Mary L. Bouxsein}

Department of Orthopedic Surgery, Harvard Medical School, Center for Advanced Orthopedic Studies, Beth Israel Deaconess Medical Center, Boston, MA

\# These authors contributed equally to this work.

\section{Abstract}

Background: Although dual-energy x-ray absorptiometry (DXA) assessed areal bone density (aBMD) is the clinical standard for determining fracture risk, the majority of older adults who sustain a fracture do not have osteoporosis (T-score < -2.5 ). Importantly, bone fragility results not only from low BMD, but also from deterioration in bone structure. We used high-resolution peripheral quantitative computed tomography (HR-pQCT) data from eight cohorts to evaluate whether HR-pQCT indices were associated with fracture risk independently of femoral neck (FN) aBMD and FRAX (Fracture Risk Assessment Tool) score.

Methods: Participants included 7,254 individuals (66\% women) from cohorts in the USA (Framingham, Mayo Clinic), France (QUALYOR, STRAMBO, OFELY), Switzerland (GERICO), Canada (CaMos), and Sweden (MrOS). We used Cox proportional hazards models to estimate 
hazards ratios (HRs) for the association between bone parameters (per standard deviation, SD, deficit) and incident fracture, adjusting for age, sex, height, weight and cohort.

Findings: Mean baseline age was $69( \pm 9)$ years (range, 40 to 96$)$. Cumulative incidence of fracture was $11 \%(n=765)$ over a mean follow-up time of $4.6( \pm 2.4)$ years. The majority of participants $(92 \%)$ had a femoral neck T-score >-2.5, and thus did not meet diagnostic criteria for osteoporosis. Failure load was the bone measure most strongly associated with risk of fracture: tibia HR=2.40 (1.98-2.91), radius HR=2.13 (1.77-2.56), per SD decrease in failure load. HRs for other bone indices ranged from $\mathrm{HR}=1.12(1.03-1.23)$ per SD increase in tibia cortical porosity to $\mathrm{HR}=1.58$ (1.45-1.72) per SD decrease in radius trabecular volumetric bone density (vBMD). After further adjustment for FN aBMD or FRAX, HRs were attenuated, but most bone parameters remained significantly associated with fracture. Cortical density, trabecular number, and trabecular thickness at the distal radius were the best set of predictors of fracture; while the same indices plus cortical area were identified for the tibia. These HR-pQCT indices and failure load improved prediction of fracture, beyond FN aBMD alone or FRAX.

Interpretation: Results from this large international cohort of women and men confirm prior studies showing that deficits in trabecular and cortical bone density and structure contribute to fracture risk independently of aBMD and FRAX. Measurements of cortical and trabecular bone density and morphology at the peripheral skeleton may improve identification of those at highest risk for fracture.

Funding: National Institutes of Health, National Institute of Arthritis Musculoskeletal and Skin Diseases, R01AR061445

\section{Introduction}

Fragility fractures, which lead to significant morbidity, mortality and expense, are a large public health concern. Notably, the number of women who will experience a fracture in any given year exceeds the combined number who will experience a new stroke, breast cancer or myocardial infarction. Annual costs associated with fragility fractures exceeds $\$ 19$ billion in the US and 36 billion euros in Europe ${ }^{1}$. Given the predicted growth in the number of older adults, fractures and associated costs are projected to increase two- to four-fold worldwide in the next few decades. Despite this, osteoporosis remains considerably underdiagnosed and undertreated ${ }^{2}$.

Current approaches to osteoporosis diagnosis and fracture risk prediction rely on casefinding strategies based on bone mineral density (BMD) measurements by dual-energy Xray absorptiometry (DXA), combined with clinical risk factors that have been incorporated into the FRAX algorithm. However, the majority of fractures occur in individuals not diagnosed with osteoporosis by BMD testing and/or in those with few clinical risk factors, and thus low fracture probability by FRAX (Fracture Risk Assessment Tool ${ }^{3}$. Improved methods to identify those at highest risk for fracture would allow for treatment of patients who would likely have the greatest benefit-to-risk profile, and may ultimately reduce fracture burden.

Whole bone strength is determined not only by bone mass, but also by bone morphology and microarchitecture. As DXA-based BMD measurements are limited to assessment of bone 
mass only, other techniques that assess bone morphology and/or microstructure may contribute to fracture risk prediction. Trabecular bone score, a 2D textural measured derived from a posteroanterior lumbar spine DXA scan, slightly improves prediction of hip fracture risk compared to BMD alone, but does not improve assessment of vertebral or major osteoporotic fractures ${ }^{4}$. Computed tomography is able to assess cortical and trabecular bone at central skeletal sites, but lacks the resolution to provide information about bone microstructure. High-resolution peripheral computed tomography (HR-pQCT), introduced over a decade ago, is able to assess cortical and trabecular bone density and microarchitecture at peripheral skeletal sites, with minimal radiation exposure. Many studies have reported that trabecular and cortical bone microstructure and estimated failure load assessed from HR-pQCT scans can discriminate those individuals with a history of fracture from non-fracture controls, often independently of DXA-BMD ${ }^{5-10}$.

In addition, a few studies have recently demonstrated that bone measurements from HRpQCT can predict incident fractures in men ${ }^{11-13}$ and women ${ }^{14,15}$ independently of DXABMD and in some cases, FRAX. Yet, these studies were limited by relatively few incident fractures (71 to 135), and no studies included both women and men.

Thus, the objective of this prospective multi-national cohort study, was to determine whether peripheral bone density and microstructure predict incident fracture in older women and men, and whether they do so independently of femoral neck DXA-BMD and FRAX. We selected DXA-BMD of the femoral neck, because this is the site used clinically for fracture prediction as well as employed by FRAX. In addition, we aimed to determine the combination of HR-pQCT variables that were most strongly associated with incident fracture. Based on the prior studies ${ }^{11,14-16}$, our primary hypothesis was that lower trabecular bone density, cortical area and failure load (from micro-finite element analyses) at both the radius and tibia would be associated with incident fracture independent of femoral neck BMD and FRAX.

\section{Methods}

\section{Participants and study design}

Individuals in this study are members of eight cohorts comprising the Bone Microarchitecture International Consortium, or BoMIC, including the Framingham Study ${ }^{17}$, Qualyor (Qualite Osseuse Lyon Orleans) ${ }^{18}$, GERICO (Geneva Retirees Cohort) ${ }^{19}$, CaMos (Canadian Multicentre Osteoporosis Study) ${ }^{20}$, STRAMBO (Structure of Aging Men's Bones) $)^{21}$, OFELY (Os des Femmes de Lyon) ${ }^{22}$, MrOS (Osteoporotic Fractures in Men Study) Sweden ${ }^{23}$, and Mayo Clinic ${ }^{24}$ (Table 1). Each of the BoMIC cohorts was established to study risk factors for osteoporosis, conducting comprehensive clinical examinations, administering standardized questionnaires, and prospectively ascertaining incident fractures.

Participants in the current study included 7,254 individuals (4,768 women, 2,486 men) who underwent HR-pQCT scanning and were at least 40 years of age. Follow-up time was defined as the years contributed by each participant from baseline (HR-pQCT scanning) to the first of the following events: fracture, death, last contact, or closing date (specific for 
each cohort). Participants in each cohort provided written informed consent, and the Institutional Review Board for Human Research at Hebrew SeniorLife approved the study.

\section{Incident fracture}

We defined incident fracture as the first fracture occurring subsequent to HR-pQCT. Incident fractures included both non-traumatic and traumatic fractures, with the exception of the skull, face, sternum, finger, and toe and pathological fracture. We also excluded incident non-clinical spine fractures since few cohorts performed serial radiologic evaluations; thus, we included only incident clinical spine fractures. Across the cohorts, methods to ascertain fractures included a combination of self-report by participants and identification through medical records and confirmation by medical professionals or trained research staff. We considered as a primary outcome all incident fractures (or 'any' incident fractures), and as a secondary outcome "major osteoporotic fractures", defined as incident fractures of the proximal humerus, wrist, distal forearm, clinical spine, or hip ${ }^{25}$.

\section{High-resolution peripheral quantitative computed tomography (HR-pQCT)}

Volumetric bone density and bone microarchitecture were assessed at the ultra-distal tibia and ultra-distal radius using HR-pQCT (XtremeCT, Scanco Medical AG, Switzerland). HRpQCT scans were acquired with a nominal isotropic voxel size of $82 \mu \mathrm{m}^{3}$. Scanning was performed on the non-dominant forearm and leg (on the same side), with the exception of the Framingham Study which always scanned the right leg, and the CaMos and MrOS Sweden cohorts which always scanned the left leg. If a participant reported previous extremity fracture or had metal in the region of the scan, then the contralateral extremity was examined. Antero-posterior scout views were used to place a reference line on the distal tibial and radial joint surfaces, as previously described ${ }^{26}$. The scan region (110 slices) was 9 $\mathrm{mm}$ in length and offset proximally to the reference line by $22.5 \mathrm{~mm}$ for the tibia and 9.5 $\mathrm{mm}$ for the radius. Scans were evaluated for motion artifacts and repeated if significant movement occurred. ${ }^{27}$ Scanning of a quality control phantom, containing rods of hydroxyapatite (densities of $0,100,200,400$ and $800 \mathrm{mg} \mathrm{HA} / \mathrm{cm}^{3}$ ), was performed daily to monitor longitudinal stability of the system.

At each clinical site, scans were graded using a 5-point movement artifact scale (1=none, $2=$ minor, $3=$ moderate, $4=$ severe, and $5=$ extreme $)^{27}$. For density measures, scans with movement artifact rated grades 1 to 4 were retained, but for microarchitectural measures, only grades 1 to 3 were retained. A standard analysis program (Scanco software version V6.0) was used to assess total cross-sectional area, total density, trabecular density, and trabecular microarchitecture, and a semi-automated cortical bone segmentation technique to assess cortical density and cortical microarchitecture ${ }^{28}$.

The central coordinating site reviewed the data from each cohort for outliers and consistency. All sites used the same scanner, and operators were trained by a single manufacturer and followed the same protocol for scan acquisition and analysis. 


\section{Micro-finite element analysis}

Linear micro-finite element analysis ( $\mu$ FEA; IPLFE, Scanco Medical AG for OFELY, GERICO and MrOS Sweden; Numerics88 Solutions Inc. for CaMos, Framingham, and Mayo Clinic) was performed to estimate failure load (N: Newtons), as previously described ${ }^{29}$. Briefly, axial compression conditions were applied with $1 \%$ apparent strain. Failure load was defined as the load when $2 \%$ of the elements exceeded a strain of 0.007 . Boundary conditions used in cohorts were either axial or uniaxial loading, and a range of tissue moduli were used ( 6.829 to $20 \mathrm{GPa}$ ). Therefore, harmonization of the failure load across cohorts was achieved by linearly calibrating to approximate axial conditions with a tissue modulus of $6.829 \mathrm{GPa}$. The calibration curves were generated by solving a large cohort $(\mathrm{N}=1,371)$ with variations of boundary conditions and tissue moduli ${ }^{30}$. The RMS estimated error for calibration is between 1.82 to $3.99 \%$. Micro-finite element analysis was not performed in QUALYOR ( $\mathrm{N}=1,504$ women) and STRAMBO (810 men), and 277 tibia scans and 649 radius scans were excluded due to motion artifact.

\section{Areal BMD by DXA}

Areal BMD at the femoral neck (FN aBMD) and ultra-distal radius was assessed using Hologic DXA scanners in Qualyor, Gerico, Strambo, OFELY, and MrOS Sweden. The Framingham and Mayo Clinic cohorts used Lunar DXA, and CaMos used both Hologic and Lunar scanners depending on the clinical site. We standardized FN aBMD values and calculated T-scores using published equations ${ }^{31}$. Our primary analysis evaluated whether HR-pQCT indices predicted fracture, independent of FN aBMD, because this site is used clinically for evaluation of osteoporosis.

In a supplementary analysis, we explored whether associations between radius HR-pQCT indices and incident fracture persisted after adjustment for DXA aBMD in the same ultradistal radius site as the HR-pQCT measures. DXA radius aBMD was not performed at the time of HR-pQCT scanning in Camos ( $\mathrm{n}=838)$ and MrOs Sweden $(\mathrm{n}=491)$, and 182 individuals from other cohorts were missing data on DXA radius aBMD.

\section{FRAX (Fracture Risk Assessment Tool) for Major Osteoporosis Fracture}

Each cohort provided FRAX for major osteoporosis related fracture (and FRAX for hip fracture) calculated with FN aBMD ${ }^{25}$. FRAX calculates ten-year fracture probability, specific to country and race, using twelve clinical risk factors (age, sex, weight, height, previous fracture, parent fractured hip, current smoking, glucocorticoids, rheumatoid arthritis, secondary osteoporosis, alcohol consumption, and FN aBMD, assessed by DXA). Age, sex, weight, and height are required to calculate FRAX. If information is not available for any other 'inputs', for example, previous fracture or parent fractured hip, the algorithm assumes a response of 'no' for these items ${ }^{25}$. The cohorts did not provide the distribution of missing data for variables used in the FRAX calculation. FRAX for major osteoporosis related fracture was used in analyses.

\section{Covariates}

Each cohort provided information on covariates obtained at the time of the HR-pQCT scanning. Weight and height were measured using standardized methods. Body mass index 
(BMI) was calculated as weight $(\mathrm{kg})$ divided by height $(\mathrm{m})$ squared. Information on age, smoking, alcohol consumption, and use of osteoporosis medication was obtained using questionnaires. History of fracture during adulthood was ascertained using a combination of data collected via questionnaire and active surveillance in each cohort. Prior fracture included non-traumatic and traumatic fracture throughout adulthood, excluding fracture of the skull, finger, toe, hand, foot, and ankle, and pathologic fracture.

\section{Statistical analysis}

We considered two fracture outcomes (described above) in our statistical analysis. Our primary outcome included all incident fractures, and a secondary outcome was restricted to major osteoporotic fractures.

We used Cox proportional hazard regression models to calculate hazard ratios (HRs) and 95\% confidence intervals (CIs) for the association between individual bone traits and incidence of fracture. We conducted the analysis using individual level data for all cohorts with the exception of MrOS Sweden, where individual level data were not available. Analyses were performed separately for MrOS Sweden, and then we performed metaanalyses using cohort level data.

We standardized each bone parameter to standard deviation (SD) units, and expressed HRs per one unit change in the expected direction of increased fracture risk. We defined a set of "base covariates" to include age, sex, cohort, height, and weight. To evaluate whether HRpQCT indices predicted fracture independently from FN aBMD, we compared results from a model with adjustment for the base covariates to a model that included the base covariates plus FN aBMD. To evaluate whether HR-pQCT indices predicted fracture independently from FRAX (for major osteoporotic fracture), we compared a model adjusted only for cohort to a model adjusted for cohort and FRAX, since FRAX takes into account age, sex, height, and weight.

We performed two secondary analyses. First, we repeated our analyses in women and men separately to provide sex-specific results. Second, we used a model adjusting for radius aBMD to evaluate whether radius HR-pQCT indices predicted fracture independently from DXA aBMD measured in the same (radius) skeletal site as the HR-pQCT indices. Because data on DXA radius aBMD was not complete for all the cohorts (see methods above: Areal BMD by DXA), we considered this analysis as exploratory.

We used the following approach to select the set of HR-pQCT bone measures that best predicted incident fracture, including all cohorts in the sample, with the exception of MrOS Sweden. First, we identified the bone indices nominally associated with incident fracture in bivariate analysis. Second, we performed correlation analysis among these bone measures and selected variables that were not strongly correlated with each other (i.e., $r<0.80$ ). Third, we used a variance inflation factor (VIF) to further assess potential multicollinearity and excluded bone measures where VIF was greater than 30. Finally, we used a best subsets selection procedure (global score statistic) and Akaike Information Criterion (AIC) to select the bone measures for a final model. We used Harrell's concordance statistic to quantify the predictive ability of models. ${ }^{32}$ We used a bootstrap method, using 2,000 samples, to estimate 
standard errors and construct confidence intervals to test differences in the predictive ability of models. Third, we compared the predictive accuracy of models including the "best set" of HR-pQCT parameters plus FN aBMD, to models with FN aBMD alone and models with FRAX alone. Finally, we compared the predictive accuracy of models with $\mu$ FEA failure load to models with FN aBMD alone.

We performed four secondary analyses. First, we calculated sex-specific HRs (and 95\% CIs) for the association between individual bone traits and incident fracture. Second, we used a model adjusting for radius aBMD to evaluate whether radius HR-pQCT indices predicted fracture independently from aBMD measured in the same skeletal site. Third, we compared the predictive accuracy of models including the best set of HR-pQCT parameters plus radius aBMD, to models with radius aBMD alone, and we compared models including $\mu \mathrm{FEA}$ failure load plus radius aBMD to models with radius aBMD alone. Fourth, we performed a Net Reclassification Improvement (NRI) analysis to compare models that included the best set of HR-pQCT measures plus FN aBMD, to models with FN aBMD alone, and also to compare models with $\mu$ FEA failure load plus FN aBMD, to models with femoral neck aBMD alone. We used Cox-proportional hazards models to generate predicted probabilities of fractures ("events") at 5 years and estimate continuous (category-free) NRI.

\section{Role of the funding source}

The funder of the study had no role in study design, data collection, data analysis, data interpretation, or writing of the report. The corresponding author had full access to all the data in the study and had final responsibility for the decision to submit for publication.

\section{Results}

Participants in the current study included $\mathrm{N}=7,254$ individuals (4,768 women, 2,486 men), who had an HR-pQCT scan at the radius or tibia and were followed for incident fracture for a mean $( \pm \mathrm{SD}) 4.63$ ( \pm 2.41 ) years (range, 0.01 to 11.04 years); Table 1. Mean age was 69 $( \pm 9)$ years (range, 40-96). Eleven percent of participants used osteoporosis medications, and $23 \%$ had prior fractures. Mean FRAX score was $2 \%( \pm 4)$ for hip fracture and $9 \%( \pm 6)$ for major osteoporosis fracture. More than half of participants (54\%) had a femoral neck Tscore in the osteopenia range (between -2.5 to -1.0$), 38 \%$ in the normal range ( $\geq-1.0)$, and $8 \%$ in the osteoporosis category $(\leq-2.5)$.

The incidence rate of fracture was $22.54 / 10^{3}$ person-years. Cumulative incidence was $11 \%$ (765/7,254): $12 \%(551 / 4,768)$ in women and $9 \%$ in men $(214 / 2,486)$. Fractures included $20 \%$ (150) wrist, $16 \%$ (122) spine, 12\% (92) rib, 9\% (72) ankle, 9\% (68) hip, 8\% (63) proximal humerus, $26 \%$ (198) other. The majority of incident fractures were due to a fall from standing height or less $(67 \%, n=509)$, while $19 \%(n=145)$ were attributed to a fall from greater than standing height or due to trauma. The degree of trauma was unknown for $15 \%$ $(\mathrm{n}=111)$ of fractures. Cumulative incidence of major osteoporotic fracture was $6 \%(\mathrm{n}=403)$, or an incident rate of $11.88 / 10^{3}$ person-years.

Those with incident fracture were more likely to be older, female, have lower BMI, greater frequency of osteoporosis medication use, prior fracture, T-score $<-2.5$, and higher FRAX 
score (Table 2). Unadjusted analyses showed those individuals who sustained an incident fracture had worse bone measures for nearly all parameters compared to those who did not fracture (Supplementary Table 1). In women, most of the fractures occurred in those with a baseline femoral neck T-score in the osteopenic range (64\%), with $17 \%$ of fractures occurring in those with osteoporosis, and 19\% occurring in those with normal FN aBMD Tscores (Table 2).

The HR for the association between FN aBMD (per SD decrease) and incident fracture (adjusted for age, sex, cohort, height, and weight) was 1.57 (95\% CI: 1.42-1.74); Figure 1. (HRs are expressed per SD deficit in the bone measure.) The strongest association with incident fracture was provided by the $\mu$ FEA-estimated failure load at the distal tibia, $\mathrm{HR}=2.40$ (1.98-2.91), and distal radius, $\mathrm{HR}=2.13$ (1.77-2.56). Risk of fracture was increased between $10 \%$ and $60 \%$ (per SD) for other bone indices at the tibia and radius. For example, HRs ranged from $H R=1.12$ (1.03-1.23) for tibia cortical porosity to $H R=1.58(1.45-1.72)$ for radius trabecular vBMD. In contrast, total area was not associated with incident fracture (not shown): tibia, $\mathrm{HR}=0.88$ (0.77-1.00) and radius, $\mathrm{HR}=1.01(0.87-1.16)$. The HR for the association between FRAX (per SD) and incident fracture was 1.40 (1.32-1.48).

After additional adjustment for FN aBMD, HRs were attenuated but remained significant for most parameters (Figure 1). In particular, failure load remained most strongly associated with fracture, with an HR of 1.98 (1.58-2.49) for the distal tibia and $1.82(1.47-2.25)$ for the distal radius. HRs for other indices, after adjustment for $\mathrm{FN}$ aBMD, ranged from $\mathrm{HR}=1.09$ (1.04-1.14) for tibia SD trabecular number (not shown) to $\mathrm{HR}=1.44$ (1.31-1.59) for radius trabecular vBMD. In contrast, tibia cortical thickness and trabecular thickness, and radius cortical $\mathrm{VBMD}$ and cortical tissue mineral density were no longer associated with fracture after adjustment for $\mathrm{FN}$ aBMD.

After adjusting for cohort and FRAX, HRs remained significant for all parameters at both the tibia and radius, except for cortical porosity and total area, which were weakly or not associated with fracture risk (Figure 1). HRs continued to be greatest for failure load at both the tibia and radius $(\mathrm{HR}=1.78, \mathrm{CI}=1.50-2.12$ for tibia, $\mathrm{HR}=1.76, \mathrm{CI}=1.48-2.09$ for radius), followed by $\mathrm{HRs}$ for trabecular vBMD (radius $\mathrm{HR}=1.51, \mathrm{CI}=1.38-1.65$; tibia $\mathrm{HR} 1.32$, CI 1.21-1.44), total vBMD (radius $\mathrm{HR}=1.43, \mathrm{CI}=1.31-1.56$; tibia $\mathrm{HR}=1.41, \mathrm{CI}=1.29-1.54$ ), trabecular number (radius, $\mathrm{HR}=1.39, \mathrm{CI}=1.27-1.51$; tibia $\mathrm{HR}=1.19, \mathrm{CI}=1.09-1.30$ ), and cortical area (radius $\mathrm{HR}=1.37, \mathrm{CI}=1.23-1.52$; tibia, $\mathrm{HR}=1.38, \mathrm{CI}=1.25=1.53$ ).

Restricting the outcome to incident major osteoporotic fractures (Supplementary Figure 1), we found the interpretation of results nearly identical to those for all fractures. In general, HRs for major osteoporotic fractures were either similar or somewhat stronger than HRs for all fractures. After additional adjustment for FN aBMD, HRs were attenuated but remained significant for all radius parameters and most tibia measures (exceptions included tibia cortical tissue mineral density, SD of trabecular number, and trabecular separation which were not significant after adjustment for FN aBMD).

In sex-stratified analyses, we found that results for incident fracture were largely similar in women and men (Supplementary Table 2), although effect sizes were somewhat attenuated 
in men. In secondary analyses, in which we adjusted for DXA radius aBMD (rather than FN $\mathrm{aBMD})$, we found radius trabecular number $(\mathrm{HR}=1.18,1.04$ to 1.33$)$ and radius trabecular vBMD (HR=1.26, 1.09-1.46) were independently associated with fracture risk;

Supplementary Table 3. Whilst no other radius HR-pQCT measures were statistically significantly associated with incident fracture after adjustment for DXA aBMD at the ultradistal radius, confidence intervals for most other indices were in the range 0.9-1.2.

Our model selection process identified cortical density, trabecular number, and trabecular thickness as the best set of non-collinear predictors at the distal radius; while the same variables plus cortical area were identified for the tibia. Further, these sets of predictors improved the ability to predict fracture beyond FN aBMD and FRAX (Table 3). For example, the AUC improved from 0.670 (0.646-0.693) for FN aBMD alone to 0.688 (0.665-0.711) for FN aBMD plus radius cortical density, trabecular number and trabecular thickness. Similarly, AUCs improved from 0.657 (0.634-0.679) for FRAX alone to 0.683 (0.659-0.706) for FRAX plus radius cortical density, trabecular number and trabecular thickness. Addition of $\mu$ FEA-failure load for both the distal radius and tibia also improved the AUC over that of FN aBMD alone (Table 4). Repeating this analysis for the major osteoporotic fracture outcome yielded AUCs of larger magnitude than those estimated for all incident fractures (Supplementary Tables 4-5). The best set of predictors improved the ability to predict major osteoporotic fracture beyond $\mathrm{FN}$ aBMD, at the radius ( $<<0.01$ ), but not at the tibia $(\mathrm{p}=0.11)$; Supplementary Table 4. Models for major osteoporotic fracture with $\mu$ FEA-failure load at the radius $(\mathrm{p}=0.12)$ and tibia $(\mathrm{p}=0.09)$ did not improve the AUC over that of FN aBMD ( $\mathrm{p}=0.09$ tibia, and $\mathrm{p}=0.13$ radius failure load; Supplementary Table 5).

Compared to AUCs for DXA aBMD at the ultra-distal radius, fracture prediction did not did not significantly improve with consideration of the best set of radius HR-pQCT parameters $(\mathrm{p}=0.10)$ or radius failure load $(\mathrm{p}=0.58)$; Supplementary Table 6. A supplementary NRI analyses comparing the best set of HR-pQCT indices to FN aBMD alone yielded NRIs of $11 \%$ for events (fractures), $7 \%$ for non-events, and an overall NRI of 0.18 (0.11-0.26) for the tibia, and for the radius, $7 \%$ for events, $14 \%$ for non-events, and an overall NRI of 0.21 (0.13- 0.30); Supplementary Table 7. Comparing $\mu$ FEA-failure load to FN aBMD alone resulted in NRIs of $14 \%$ for events, $3 \%$ for non-events, and overall an NRI was $0.18(0.07-$ 0.28 ) for the tibia, and $17 \%$ for events, $2 \%$ for non-events, and an overall NRI of $0.19(0.08$ 0.29 ) for the radius.

\section{Discussion}

A large number of fractures occur in individuals not identified at high risk by BMD testing and/or clinical risk factors. In this large multi-national cohort of women and men followed for 4.6 years, we found that trabecular and cortical bone density and microstructure, as well as estimated failure load measured at the peripheral skeleton, predict incident fractures independently of femoral neck BMD and FRAX. The current study included more than 7,000 participants with 765 fractures, and as such, represents the largest prospective study of HR-pQCT indices and incident fracture to date. 
Our findings are consistent with prior cross-sectional studies, as well as recent prospective studies in smaller cohorts ${ }^{11-15}$ that have demonstrated associations between cortical and trabecular bone measures at the distal radius and tibia and fracture. Notably, among the prior prospective studies, several parameters have been consistently strongly associated with the incidence of fracture, including trabecular bone density at the distal radius ${ }^{14,15}$, cortical area at the distal radius and distal tibia ${ }^{11,14}$, and stiffness or failure load, as estimated by microfinite element analysis $11,14,15$. Our current results also showed estimated failure load at the tibia and radius to be the strongest predictor of incident fracture, independently of femoral neck aBMD and FRAX. Failure load estimated by finite element analysis intrinsically includes information on structure and density and thus performs as well or better than the best combination of individual parameters. In the present study, we found additional important predictors of fracture, including cortical density, trabecular number, and trabecular thickness for both the tibia and radius. Consistent with prior work ${ }^{11,14}$, the best set of fracture predictors in our study also included cortical area at the tibia. Further, these HRpQCT variables and $\mu$ FEA-derived failure load improved the ability to predict incident fracture beyond FN aBMD and FRAX.

In contrast to some prior cross-sectional studies ${ }^{10,33}$, we did not find a strong association between cortical porosity and incident fracture. This discrepancy may be due to differences in fracture outcomes, as these previous studies evaluated forearm ${ }^{33}$ or hip ${ }^{10}$ fracture, whereas the primary fracture outcome in this study was "any" fracture and secondarily, major osteoporotic fractures. Alternatively, this discrepancy may be due to use of different approaches to assess cortical porosity. The cohorts in the current study used the thresholdbased method for porosity assessment implemented in the Scanco software, whereas prior case-control studies ${ }^{8,33}$ used the density-based method implemented in the Strax software. ${ }^{34}$ Importantly, both approaches are strongly associated $\left(\mathrm{r}^{2}>0.93\right)$ with cortical porosity as measured by gold-standard synchrotron micro-computed tomography measurements ${ }^{35}$. The threshold-based method for porosity assessment consistently underestimates, while the density-based method consistently overestimates, the "true" porosity. In addition, the algorithms used to delineate the cortical region are markedly different between the Scanco and Strax software, making direct comparisons across studies difficult.

Results from this study have important clinical implications. Notably, the majority of study participants had femoral neck BMD T-scores in the osteopenic or normal range, indicating that our results are likely valid in this important group, in whom fractures are common but standard clinical testing does not identify as high risk. In women, nearly two-thirds of fractures occurred in those with osteopenia (by DXA-femoral neck BMD). Thus, assessment of bone microstructure may be useful in those who would not otherwise be identified as being at risk for fracture. We focused on the ability of HR-pQCT to predict fracture independently of DXA aBMD at the femoral neck, the site used for clinical diagnosis of osteoporosis and determination of fracture risk. However, the femoral neck is distinct from the sites of the HR-pQCT measurements. To gain further insight into the contribution of bone microarchitecture independent of aBMD, we performed supplementary analyses adjusting for DXA aBMD at the same ultra-distal radius site as the HR-pQCT measurements and found that trabecular vBMD and trabecular number remained independent predictors of fracture. This result suggests that bone microarchitecture in the peripheral skeleton provides 
unique information about skeletal fragility not captured by DXA aBMD. However, the reduced sample size, due to missing data on DXA aBMD at the ultra-distal radius for some cohorts, compels cautious interpretation of these results.

This study has several strengths. First, a major strength of this study is the inclusion of a large sample of a non-clinical population of women and men from several countries. However, our study was limited to European and North American cohorts with few nonwhite individuals. Second, the active surveillance and adjudication of fractures by all cohorts was another important strength of this study and allowed evaluation of both incident fractures and incident major osteoporotic fractures. Third, all the cohorts used similar, standardized methods for data collection, including HR-pQCT and DXA imaging, FRAX calculation, and assessment of confounders, such as measured height and weight. We standardized FN aBMD to remove possible systematic differences between DXA systems ${ }^{31}$. Yet, we cannot entirely rule out the potential for heterogeneity in data collection across study sites. The prospective design of this study helps to ensure that heterogeneity or errors in measurement were unrelated to fracture outcomes and thus would have led to an underestimation of associations. It is also possible that information obtained by questionnaire used to calculate FRAX scores may have been subject to error. For example, individuals may have under-reported smoking and alcohol consumption thereby underestimating FRAX scores. Thus, adjustment for FRAX may have been inadequate for such individuals, possibly resulting in overestimation of associations between HR-pQCT and fracture. However, under-reporting of risk factors would also occur in clinical practice when calculating FRAX probabilities for fracture.

Our study has a few limitations. First, all of the cohorts acquired their HR-pQCT scans at a fixed distance relative to the endplate of the distal radius or tibia. Thus, the skeletal regions measured may have been slightly different anatomically, depending on the given participant's limb length and may have therefore obscured differences in bone density and morphology. A second limitation is that we did not cross-calibrate the different HR-pQCT devices used in this study. By increasing "noise" in HR-pQCT measurement, these technical limitations may have led to an underestimation of associations. Importantly, the central coordinating site reviewed the HR-pQCT data from each cohort for quality control, and all sites used the same scanner and protocol for scan acquisition and analysis. Third, microfinite element analysis was not performed in all the cohorts. Nevertheless, despite relatively wide confidence intervals, failure load was the strongest predictor of fracture. Further, we found no difference in age (68 years) or fracture incidence (10\%) between participants with and without data on failure load, although femoral neck T-score was lower in those with missing failure load values than those not missing failure load (-1.35 vs. -1.12 for radius $\mu$ FEA and -1.32 vs. -1.15 for tibia $\mu$ FEA, respectively). As individuals with missing failure load on average had lower T-scores, we may have underestimated associations between failure load and fracture incidence.

In conclusion, in this multi-national cohort study of over 7,000 older women and men, we found the peripheral measurements of cortical and trabecular bone density, microstructure and strength are associated with incident fracture independently of current clinical tools for fracture risk assessment, namely femoral neck DXA-BMD and FRAX. Notably, the majority 
of fractures occurred in those who did not have osteoporosis according to BMD T-scores, but who exhibited deficits in bone microstructure. While HR-pQCT is not widely available, our findings may prompt expansion of the clinical use of HR-pQCT and the development of alternate technologies to assess cortical and trabecular bone structure and strength. Our findings provide the groundwork to develop new models for fracture prediction, thereby improving risk stratification and reducing the public health burden of osteoporosis.

\section{Supplementary Material}

Refer to Web version on PubMed Central for supplementary material.

\section{Acknowledgements}

Research reported in this publication was supported by the National Institutes of Health (NIH) (National Institute of Arthritis Musculoskeletal and Skin Diseases [NIAMS], R01AR061445; National Heart, Lung and Blood Institute [NHLBI] Framingham Heart Study, Contract No. N01-HC-25195, HHSN268201500001I). The content is solely the responsibility of the authors and does not necessarily represent the official views of the NIH. Additional support was provided by Friends of Hebrew SeniorLife and a research grant from the Investigator Initiated Studies Program of Merck Sharp \& Dohme. The opinions expressed in this work are those of the authors and do not necessarily represent those of Merck.

\section{References}

1. Burge R, Dawson-Hughes B, Solomon DH, Wong JB, King A, Tosteson A. Incidence and economic burden of osteoporosis-related fractures in the United States, 2005-2025. J Bone Miner Res 2007;22(3):465-75. [PubMed: 17144789]

2. Khosla S, Shane E. A Crisis in the Treatment of Osteoporosis. J Bone Miner Res 2016;31(8):14857. [PubMed: 27335158]

3. Jiang X, Gruner M, Tremollieres F, et al. Diagnostic accuracy of FRAX in predicting the 10-year risk of osteoporotic fractures using the USA treatment thresholds: A systematic review and metaanalysis. Bone 2017;99:20-25. [PubMed: 28274799]

4. Martineau P, Leslie WD, Johansson H, et al. Clinical utility of using lumbar spine trabecular bone score to adjust fracture probability: The Manitoba BMD Cohort. J Bone Miner Res 2017;32(7): 1568-1574. [PubMed: 28276598]

5. Szulc P, Boutroy S, Vilayphiou N, Chaitou A, Delmas PD, Chapurlat R. Cross-sectional analysis of the association between fragility fractures and bone microarchitecture in older men: the STRAMBO study. J Bone Miner Res 2011;26(6):1358-67. [PubMed: 21611974]

6. Boutroy S, Khosla S, Sornay-Rendu E, et al. Microarchitecture and peripheral BMD are impaired in postmenopausal white women with fracture independently of total hip t-score: An international multicenter study. J Bone Miner Res 2016;31(6):1158-66. [PubMed: 26818785]

7. Stein EM, Kepley A, Walker M, et al. Skeletal structure in postmenopausal women with osteopenia and fractures is characterized by abnormal trabecular plates and cortical thinning. J Bone Miner Res 2014;29(5):1101-9. [PubMed: 24877245]

8. Bala Y, Bui QM, Wang XF, et al. Trabecular and cortical microstructure and fragility of the distal radius in women. J Bone Miner Res 2015;30(4):621-9. [PubMed: 25327362]

9. Biver E, Durosier C, Chevalley T, Herrmann FR, Ferrari S, Rizzoli R. Prior ankle fractures in postmenopausal women are associated with low areal bone mineral density and bone microstructure alterations. Osteoporos Int 2015;26(8):2147-55. [PubMed: 25851699]

10. Sundh D, Nilsson AG, Nilsson M, Johansson L, Mellstrom D, Lorentzon M. Increased cortical porosity in women with hip fracture. J Intern Med 2017;281(5):496-506. [PubMed: 28097725]

11. Ohlsson C, Sundh D, Wallerek A, et al. Cortical bone area predicts incident fractures independently of areal bone mineral density in older men. J Clin Endocrinol Metab 2017;102(2): 516-524. [PubMed: 27875059] 
12. Langsetmo L, Peters KW, Burghardt AJ, et al. Volumetric bone mineral density and failure load of distal limbs predict incident clinical fracture independent of FRAX and clinical risk factors among older men. J Bone Miner Res 2018;33(7):1302-1311. [PubMed: 29624722]

13. Szulc P, Boutroy S, Chapurlat R. Prediction of fractures in men using bone microarchitectural parameters assessed by high-resolution peripheral quantitative computed tomography-The Prospective STRAMBO Study. J Bone Miner Res 2018;33(8):1470-1479. [PubMed: 29694676]

14. Biver E, Durosier-Izart C, Chevalley T, van Rietbergen B, Rizzoli R, Ferrari S. Evaluation of radius microstructure and areal bone mineral density improves fracture prediction in postmenopausal women. J Bone Miner Res 2017;33(2)328-337. [PubMed: 28960489]

15. Sornay-Rendu E, Boutroy S, Duboeuf F, Chapurlat RD. Bone microarchitecture assessed by HRpQCT as predictor of fracture risk in postmenopausal women: The OFELY Study. J Bone Miner Res 2017;32(6):1243-1251. [PubMed: 28276092]

16. Burt LA, Manske SL, Hanley DA, Boyd SK. Lower bone density, impaired microarchitecture, and strength predict future fragility fracture in postmenopausal women: 5-Year follow-up of the Calgary CaMos Cohort. J Bone Miner Res 2018;33(4)589-597. [PubMed: 29363165]

17. Samelson EJ, Demissie S, Cupples LA, et al. Diabetes and Deficits in Cortical Bone Density, Microarchitecture, and Bone Size: Framingham HR-pQCT Study. J Bone Miner Res 2018;33(1): 54-62. [PubMed: 28929525]

18. Chapurlat R, Pialat JB, Merle B, et al. The QUALYOR (QUalite Osseuse LYon Orleans) study: a new cohort for non invasive evaluation of bone quality in postmenopausal osteoporosis. Rationale and study design. Arch Osteoporos 2017;13(1):2. [PubMed: 29282548]

19. Pepe J, Biver E, Bonnet N, et al. Within- and Across-Sex Inheritance of Bone Microarchitecture. J Clin Endocrinol Metab 2017;102(1):40-45. [PubMed: 27732327]

20. Nishiyama KK, Macdonald HM, Hanley DA, Boyd SK. Women with previous fragility fractures can be classified based on bone microarchitecture and finite element analysis measured with HRpQCT. Osteoporos Int 2013;24(5):1733-40. [PubMed: 23179565]

21. Chaitou A, Boutroy S, Vilayphiou N, et al. Association between bone turnover rate and bone microarchitecture in men: the STRAMBO study. J Bone Miner Res 2010;25(11):2313-23. [PubMed: 20499368]

22. Sornay-Rendu E, Boutroy S, Munoz F, Delmas PD. Alterations of cortical and trabecular architecture are associated with fractures in postmenopausal women, partially independent of decreased BMD measured by DXA: the OFELY study. J Bone Miner Res 2007;22(3):425-33. [PubMed: 17181395]

23. Mellstrom D, Johnell O, Ljunggren O, et al. Free testosterone is an independent predictor of BMD and prevalent fractures in elderly men: MrOS Sweden. J Bone Miner Res 2006;21(4):529-35. [PubMed: 16598372]

24. Amin S, Khosla S. Sex- and age-related differences in bone microarchitecture in men relative to women assessed by high-resolution peripheral quantitative computed tomography. J Osteoporos 2012;2012:129760. [PubMed: 22496983]

25. Kanis JA, Oden A, Johansson H, Borgstrom F, Strom O, McCloskey E. FRAX and its applications to clinical practice. Bone 2009;44(5):734-43. [PubMed: 19195497]

26. Boutroy S, Bouxsein ML, Munoz F, Delmas PD. In vivo assessment of trabecular bone microarchitecture by high-resolution peripheral quantitative computed tomography. J Clin Endocrinol Metab 2005;90(12):6508-15. [PubMed: 16189253]

27. Pialat JB, Burghardt AJ, Sode M, Link TM, Majumdar S. Visual grading of motion induced image degradation in high resolution peripheral computed tomography: impact of image quality on measures of bone density and micro-architecture. Bone 2012;50(1):111-8. [PubMed: 22019605]

28. Buie HR, Campbell GM, Klinck RJ, MacNeil JA, Boyd SK. Automatic segmentation of cortical and trabecular compartments based on a dual threshold technique for in vivo micro-CT bone analysis. Bone 2007;41(4):505-15. [PubMed: 17693147]

29. Pistoia W, van Rietbergen B, Lochmuller EM, Lill CA, Eckstein F, Ruegsegger P. Estimation of distal radius failure load with micro-finite element analysis models based on three-dimensional peripheral quantitative computed tomography images. Bone 2002;30(6):842-8. [PubMed: 12052451] 
30. Whittier DE, Manske SL, Kiel DP, Bouxsein M, Boyd SK. Harmonizing finite element modelling for non-invasive strength estimation by high-resolution peripheral quantitative computed tomography. J Biomech 2018;80:63-71. [PubMed: 30201250]

31. Fan B, Lu Y, Genant H, Fuerst T, Shepherd J. Does standardized BMD still remove differences between Hologic and GE-Lunar state-of-the-art DXA systems? Osteoporos Int 2010;21(7):122736. [PubMed: 19859644]

32. Harrell FE, Jr., Lee KL, Califf RM, Pryor DB, Rosati RA. Regression modelling strategies for improved prognostic prediction. Stat Med 1984;3(2):143-52. [PubMed: 6463451]

33. Bala Y, Zebaze R, Ghasem-Zadeh A, et al. Cortical porosity identifies women with osteopenia at increased risk for forearm fractures. J Bone Miner Res 2014;29(6):1356-62. [PubMed: 24519558]

34. Zebaze R, Ghasem-Zadeh A, Mbala A, Seeman E. A new method of segmentation of compactappearing, transitional and trabecular compartments and quantification of cortical porosity from high resolution peripheral quantitative computed tomographic images. Bone 2013;54(1):8-20. [PubMed: 23334082]

35. Jorgenson BL, Buie HR, McErlain DD, Sandino C, Boyd SK. A comparison of methods for in vivo assessment of cortical porosity in the human appendicular skeleton. Bone 2015;73:167-75.

[PubMed: 25540917] 


\section{Research in Context}

\section{Evidence before this study}

When this study was planned, prior work was limited to case-control studies of fracture history and cortical and trabecular bone density and microarchitecture at peripheral skeletal sites, evaluated by high-resolution peripheral computed tomography (HR-pQCT). These initial studies showed that bone measurements from HR-pQCT discriminated between individuals with a history of fracture from non-fracture controls, often independently of areal bone mineral density (aBMD) measurements by dual-energy Xray absorptiometry (DXA). More recently, a few prospective studies have demonstrated that HR-pQCT bone indices predicted incident fractures, independently of DXA-aBMD and in some cases, FRAX (Fracture Risk Assessment Tool), an algorithm used to estimate the 10-year probability of fracture. Yet, these studies were limited by relatively few incident fractures, and no studies included both women and men.

\section{Added value of this study}

The Bone Microarchitecture International Consortium, or BoMIC, includes 7,254 individuals (4,768 women and 2,486 men) from eight cohorts in North America and Europe. Thus, the current study represents the largest prospective study of HR-pQCT indices and incident fracture to date. In this large multi-national cohort of women and men, we found that trabecular and cortical bone density and microstructure, as well as estimated failure load measured at the peripheral skeleton, predict incident fractures, independently of femoral neck aBMD and FRAX. For example, each standard deviation (SD) decrease in failure load (estimated from micro-finite element analyses) was associated with a two-fold increase in risk of fracture, after accounting for age, sex, height, weight, and femoral neck aBMD or FRAX. Cortical density, cortical area, trabecular number, and trabecular thickness were the best set of fracture predictors, each parameter associated with a $20 \%$ to $50 \%$ increased fracture risk per SD decrease in HRpQCT measure. Importantly, most study participants had femoral neck aBMD T-scores in the osteopenic or normal range, indicating that our results are applicable to this important group.

\section{Implications of all the available evidence}

Our results demonstrate that assessment of bone microstructure may be useful in those who would not otherwise be identified as being at risk for fracture and highlight the importance of compartment specific assessments of bone density and microstructure in evaluating skeletal fragility in older adults. Whilst femoral neck aBMD and FRAX remain the current clinical standards for risk stratification, this work provides a critical 'first step' in identifying additional bone traits that may add to prediction of fracture risk. 


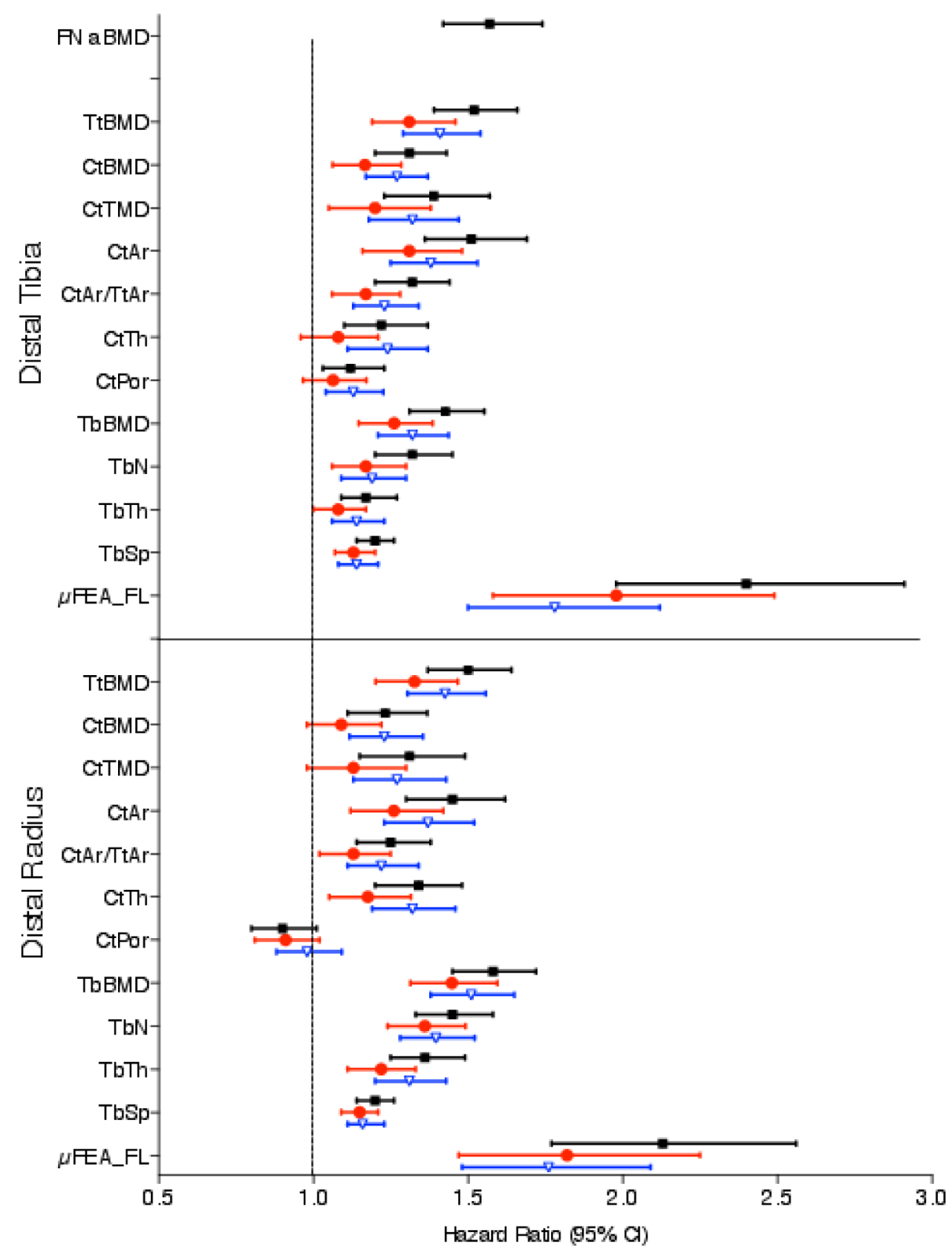

Figure 1.

Hazard ratios (HR) and 95\% confidence intervals (CI) represent associations between femoral neck (FN) aBMD (DXA) and HR-pQCT indices at the tibia (top) and radius (bottom) and incidence of any fracture. HRs are expressed per one standard deviation change in the bone measure in the expected direction of increased fracture risk. Black squares indicate HRs adjusted for age, sex, height, weight and cohort. Red circles indicate HRs additionally adjusted for FN aBMD. Blue inverted triangles indicate HRs adjusted for cohort and FRAX score (for major osteoporotic fracture). Cumulative incidence of (any) fracture was 765/7,254 (11\%). Numerical data are available in Supplementary Table 8. 
* Incident fracture includes all sites except skull, face, sternum, finger, and toe and pathological fracture

FN aBMD=femoral neck areal BMD (DXA), TtBMD=total (integral) volumetric bone mineral density, $\mathrm{CtBMD}=$ cortical volumetric bone mineral density, $\mathrm{CtTMD}=$ cortical tissue mineral density, $\mathrm{CtAr}=$ cortical area, $\mathrm{CtAr} / \mathrm{TtAr}=$ cortical area proportion, $\mathrm{CtTh}=$ cortical thickness, $\mathrm{CtPo}=$ cortical porosity, $\mathrm{TbBMD}=$ trabecular volumetric bone mineral density, $\mathrm{TbN}=$ trabecular number, $\mathrm{TbTh}=$ trabecular thickness, $\mathrm{TbSp}=$ trabecular separation, $\mu$ FEA_FL=Micro finite element analysis failure load 


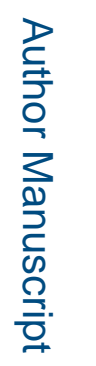

\begin{tabular}{|c|c|c|c|c|c|c|c|c|c|c|c|c|c|c|c|c|c|}
\hline . & I] & ఫ్రీ & 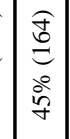 & $\left|\begin{array}{l}9 \\
+1 \\
+1\end{array}\right|$ & 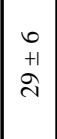 & \begin{tabular}{|l}
0 \\
6 \\
0 \\
0 \\
0 \\
-0
\end{tabular} & 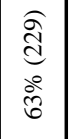 & $\mid \begin{array}{l}n \\
0 \\
+1 \\
+ \\
0 \\
0\end{array}$ & $\begin{array}{l}n \\
+1\end{array}$ & $\sigma$ & & $\begin{array}{l}\widehat{g} \\
\stackrel{g}{\sigma} \\
\text { go }\end{array}$ & 8 & $\Xi$ & & & \\
\hline of & 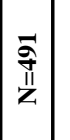 & & 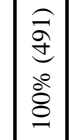 & $\left|\begin{array}{l}+ \\
+1 \\
+1 \\
\infty\end{array}\right|$ & $\begin{array}{l}m \\
+ \\
0 \\
0\end{array}$ & $\mid \begin{array}{l}\infty \\
\vdots \\
a\end{array}$ & 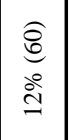 & $\mid \begin{array}{l}m \\
0 \\
+1 \\
\infty \\
0 \\
0 \\
0\end{array}$ & 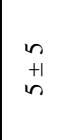 & $\begin{array}{l}0 \\
0 \\
01\end{array}$ & & 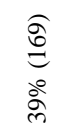 & $\frac{1}{20}$ & $\left|\begin{array}{c}\tilde{I} \\
\text { +1 } \\
\infty \\
\tilde{m}\end{array}\right|$ & $\begin{array}{l}\hat{E} \\
\text { o. } \\
\text { o. }\end{array}$ & & \\
\hline 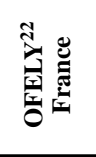 & $\begin{array}{l}\text { 政 } \\
\frac{\pi}{2}\end{array}$ & 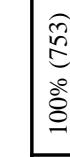 & & \begin{tabular}{|l|}
$\frac{1}{7}$ \\
+1 \\
0
\end{tabular} & 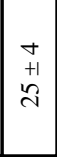 & $\mid \begin{array}{c}0 \\
0 \\
0 \\
0 \\
0\end{array}$ & 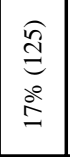 & $\mid \begin{array}{l}1 \\
0 \\
0 \\
+1 \\
0\end{array}$ & 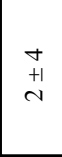 & $\begin{array}{l}\circ \\
+1\end{array}$ & & مे & $\frac{0}{0}$ & $\mid \begin{array}{c}0 \\
i \\
+1 \\
+\infty \\
\infty\end{array}$ & d & & \\
\hline$\hat{\theta}$ & $\begin{array}{l} \\
0 \\
\pi\end{array}$ & & $\left|\begin{array}{c}0 \\
\frac{0}{0} \\
\frac{0}{0} \\
\frac{0}{9}\end{array}\right|$ & $\begin{array}{l}1 \\
+1\end{array}$ & $\begin{array}{l}+ \\
+1\end{array}$ & 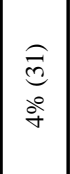 & 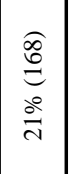 & $\left|\begin{array}{c}0 \\
0 \\
+1\end{array}\right|$ & & + & & 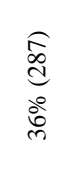 & 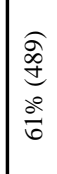 & 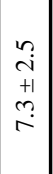 & & & \\
\hline
\end{tabular}

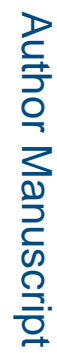

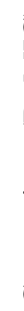

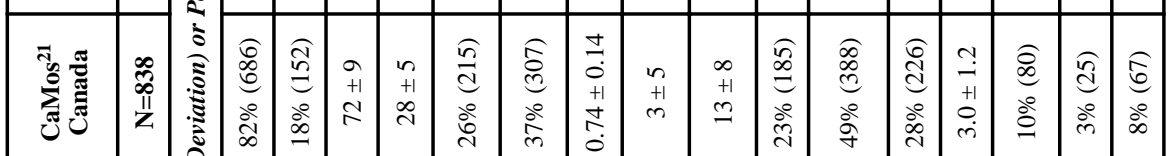

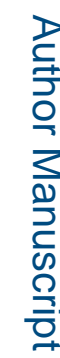
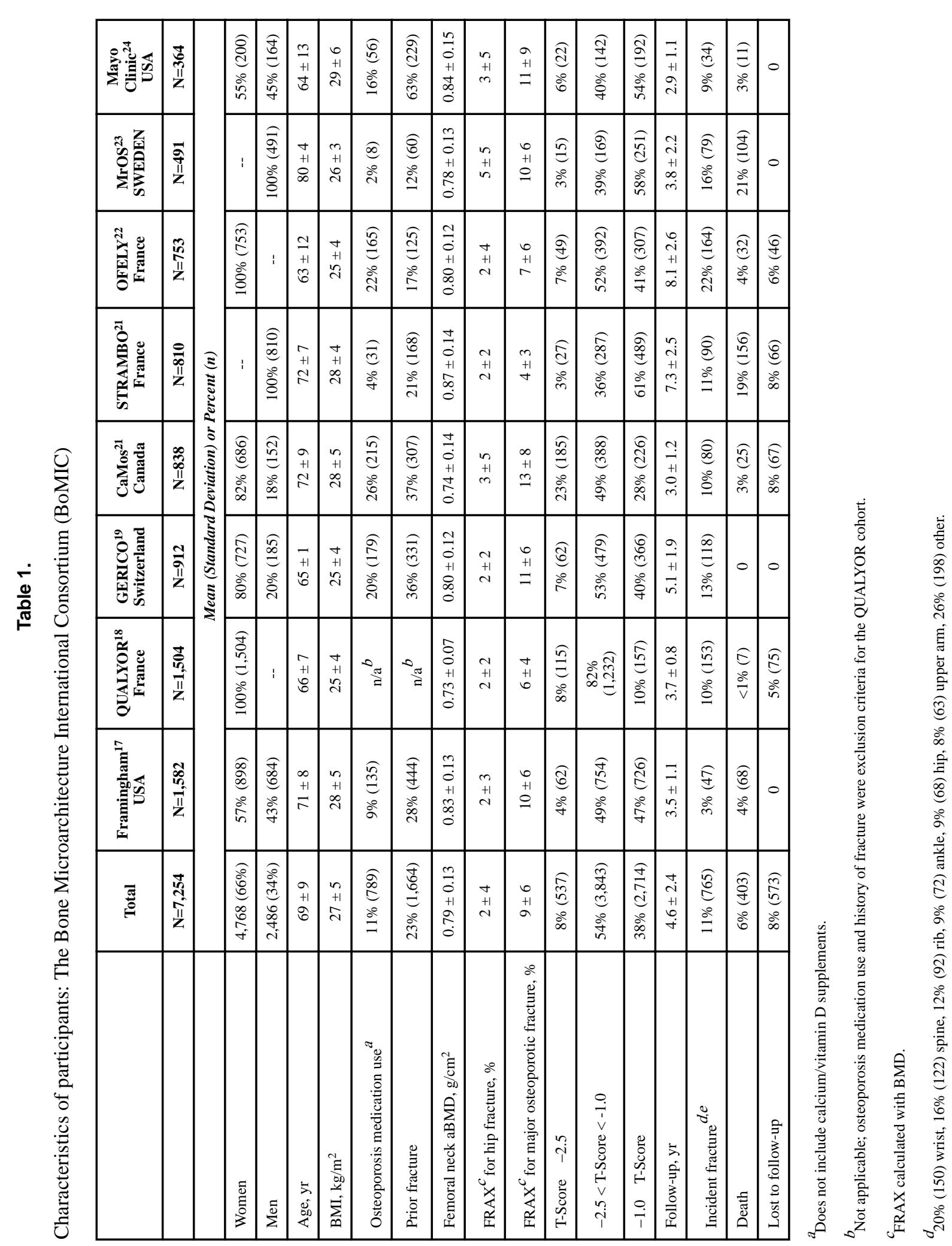

Lancet Diabetes Endocrinol. Author manuscript; available in PMC 2020 January 01. 
Table 2.

Characteristics of participants by incident fracture status

\begin{tabular}{|c|c|c|c|c|c|c|}
\hline & \multicolumn{2}{|c|}{$\begin{array}{c}\text { Total } \\
(\mathbf{N}=7,254)\end{array}$} & \multicolumn{2}{|c|}{$\begin{array}{c}\text { Women } \\
(\mathrm{N}=4,768)\end{array}$} & \multicolumn{2}{|c|}{$\begin{array}{c}\text { Men } \\
(\mathrm{N}=2,486)\end{array}$} \\
\hline & \multicolumn{2}{|c|}{ Incident Fracture } & \multicolumn{2}{|c|}{ Incident Fracture } & \multicolumn{2}{|c|}{ Incident Fracture } \\
\hline & Yes & No & Yes & No & Yes & No \\
\hline & $N=765$ & $\mathrm{~N}=\mathbf{6 , 4 8 9}$ & $N=551$ & $\mathrm{~N}=4,217$ & $\mathrm{~N}=214$ & $\mathrm{~N}=2,272$ \\
\hline & $\begin{array}{c}\text { Mean }(S D) \text { or } \\
\text { Percent (n) }\end{array}$ & $\begin{array}{c}\text { Mean }(S D) \text { or } \\
\text { Percent }(n)\end{array}$ & $\begin{array}{c}\text { Mean }(S D) \text { or } \\
\text { Percent }(n)\end{array}$ & $\begin{array}{l}\text { Mean }(S D) \text { or } \\
\text { Percent (n) }\end{array}$ & $\begin{array}{l}\text { Mean }(S D) \text { or } \\
\text { Percent }(n)\end{array}$ & $\begin{array}{c}\text { Mean }(S D) \text { or } \\
\text { Percent (n) }\end{array}$ \\
\hline Women & $72 \%(551)^{*}$ & $65 \%(4,217)$ & -- & -- & -- & -- \\
\hline Age, yr & $70(10)^{*}$ & $69(9)$ & $68(9)^{*}$ & $67(9)$ & $75(8)^{*}$ & $72(9)$ \\
\hline BMI, $\mathrm{kg} / \mathrm{m}^{2}$ & $26(5)^{*}$ & $27(5)$ & $26(5)$ & $26(5)$ & $27(4)^{*}$ & $28(4)$ \\
\hline Osteoporosis medication use & $16 \%(121)^{*}$ & $10 \%(668)$ & $20 \%(109)^{*}$ & $15 \%(620)$ & $6 \%(12)^{*}$ & $2 \%(48)$ \\
\hline Prior fragility fracture & $31 \%(234){ }^{*}$ & $22 \%(1,430)$ & $30 \%(164){ }^{*}$ & $19 \%(820)$ & $33 \%(70){ }^{*}$ & $27 \%(610)$ \\
\hline Femoral neck aBMD, $\mathrm{g} / \mathrm{cm}^{2}$ & $0.75(0.12)^{*}$ & $0.80(0.13)$ & $0.74(0.11)^{*}$ & $0.77(0.11)$ & $0.78(0.14)^{*}$ & $0.85(0.14)$ \\
\hline T-Score $\leq-2.5$ & $14 \%(103)^{*}$ & $7 \%(434)$ & $17 \%(93)^{*}$ & $9 \%(370)$ & $5 \%(10)$ & $3 \%(64)$ \\
\hline$-2.5<\mathrm{T}$-Score $<-1.0$ & $60 \%(443)^{*}$ & $53 \%(3,400)$ & $64 \%(347)^{*}$ & $64 \%(2,642)$ & $51 \%(96)^{*}$ & $34 \%(758)$ \\
\hline$-1.0<\mathrm{T}$-Score & $26 \%(190)^{*}$ & $40 \%(2,524)$ & $19 \%(106)^{*}$ & $28 \%(1,145)$ & $44 \%(84) *$ & $63 \%(1,379)$ \\
\hline FRAX for hip fracture, $\%$ & $4(5)^{*}$ & $2(4)$ & $4(5)^{*}$ & $2(4)$ & $4(6)^{*}$ & $2(3)$ \\
\hline $\begin{array}{l}\text { FRAX for major osteoporotic } \\
\text { fracture, } \%\end{array}$ & $11(8)^{*}$ & $9(6)$ & $12(9)^{*}$ & $10(7)$ & $9(7)^{*}$ & $7(4)$ \\
\hline
\end{tabular}

* Significant differences by fracture status $(\mathrm{p}<0.05)$ 
Table 3.

Predictive accuracy of models with tibia and radius HR-pQCT bone indices selected as best set of predictors of incident fracture compared to models with femoral neck aBMD or FRAX alone.

\begin{tabular}{|c|c|c|c|}
\hline & AUC (C-Statistic) & $95 \% \mathrm{CI}$ & P-Value \\
\hline \multicolumn{4}{|l|}{ Models compared to femoral neck (FN) aBMD ${ }^{*}$} \\
\hline \multicolumn{4}{|l|}{ Tibia $(\mathrm{N}=6,487)$} \\
\hline FN aBMD & 0.665 & $0.643-0.687$ & Reference \\
\hline FN aBMD + cortical area, cortical vBMD, trabecular number, trabecular thickness & 0.676 & $0.653-0.698$ & 0.0059 \\
\hline \multicolumn{4}{|l|}{ Radius $(\mathrm{N}=\mathbf{6 , 0 5 2})$} \\
\hline FN aBMD & 0.670 & $0.646-0.693$ & Reference \\
\hline FN aBMD + cortical vBMD, trabecular number, trabecular thickness & 0.688 & $0.665-0.711$ & 0.0005 \\
\hline \multicolumn{4}{|l|}{ Models compared to FRAX ${ }^{* *}$} \\
\hline \multicolumn{4}{|l|}{ Tibia $(\mathrm{N}=6,472)$} \\
\hline FRAX & 0.655 & $0.633-0.676$ & Reference \\
\hline FRAX + cortical area, cortical vBMD, trabecular number, trabecular thickness & 0.669 & $0.647-0.691$ & 0.0095 \\
\hline \multicolumn{4}{|l|}{ Radius $(\mathrm{N}=\mathbf{6 , 0 3 5})$} \\
\hline FRAX & 0.657 & $0.634-0.679$ & Reference \\
\hline FRAX + cortical vBMD, trabecular number, trabecular thickness & 0.683 & $0.659-0.706$ & 0.0002 \\
\hline
\end{tabular}

* Models adjusted for age, sex, cohort, weight and height

**

Models adjusted for cohort; FRAX incorporates age, sex, height, and weight

$\mathrm{AUC}=$ Area under the curve $\mathrm{CI}=95 \%$ confidence interval 
Table 4.

Predictive accuracy of models with tibia and radius failure load, estimated by micro-finite element analysis, compared to models with femoral neck aBMD alone.

\begin{tabular}{|c|c|c|c|}
\hline Model $^{*}$ & AUC (C-Statistic) & CI & P-Value \\
\hline Tibia (N=4,143) & & & \\
\hline FN aBMD & 0.683 & $0.654-0.712$ & Reference \\
\hline FN aBMD + failure load & 0.697 & $0.669-0.725$ & 0.0065 \\
\hline Radius (N=3,845) & & & \\
\hline FN aBMD & 0.687 & $0.657-0.717$ & Reference \\
\hline FN aBMD + failure load & 0.702 & $0.672-0.731$ & 0.0183 \\
\hline
\end{tabular}

* All models adjusted for age, sex, cohort, weight and height

$\mathrm{AUC}=$ Area under the curve $\mathrm{CI}=95 \%$ confidence interval 